\section{Periodontal infection and adverse pregnancy outcomes: a systematic review of epidemiological studies}

\author{
Infecção periodontal e desfechos \\ indesejáveis da gestação: uma revisão \\ sistemática dos estudos epidemiológicos
}

\author{
Mario Vianna Vettore 1 \\ Gabriela de Almeida Lamarca ${ }^{2}$ \\ Anna Thereza Thomé Leão ${ }^{3}$ \\ Filipe Brand Thomaz 2 \\ Aubrey Sheiham 4 \\ Maria do Carmo Leal 1
}

1 Escola Nacional de Saúde Pública Sergio Arouca, Fundação Oswaldo Cruz, Rio de Janeiro, Brasil.

2 Regional Petrópolis, Associação Brasileira de Odontologial

Seção Rio de Janeiro, Petrópolis, Brasil.

3 Faculdade de Odontologia, Universidade Federal do Rio de Janeiro, Rio de Janeiro, Brasil. ${ }^{4}$ Department of Epidemiology and Public Health, University College London, London, U. K.

\section{Correspondence} M. V. Vettore Departamento de Epidemiologia e Métodos Quantitativos em Saúde, Escola Nacional de Saúde Pública Sergio Arouca, Fundação Oswaldo Cruz. Rua Leopoldo Bulhões 1480, Rio de Janeiro, $R J$ 21041-210, Brasil. mario@ensp.fiocruz.br

\section{Abstract}

The objective of this systematic review was to evaluate analytical studies on periodontal disease as a possible risk factor for adverse pregnancy outcomes. A literature search of the MEDLINE, SciELO, and LILACS bibliographic databases and CAPES thesis database was conducted up to December 2005, covering epidemiological studies of periodontal disease and adverse pregnancy outcomes. Of the 964 papers identified, 36 analytical studies met the inclusion criteria. Twenty-six epidemiological studies reported associations between periodontal disease and adverse pregnancy outcomes. There was a clear heterogeneity between studies concerning measurement of periodontal disease and selection of type of adverse pregnancy outcome. Therefore no meta-analysis was performed. Most studies did not control for confounders, thus raising serious doubts about their conclusions. The methodological limitations of most studies did not allow conclusions concerning the effects of periodontal disease on adverse pregnancy outcomes. Larger and methodologically rigorous analytical studies using reliable outcomes and exposure measures are recommended.

Periodontal Diseases; Pregnancy; Review Literature

\section{Introduction}

The possibility that pathogenic microorganisms and their products from infectious foci (including those in the mouth) can spread to other parts of the body and trigger different diseases was first suggested by Hunter in 1910, in his "focal infection theory" 1 . The theory was criticized for its lack of sound scientific evidence and was eventually refuted. Currently, a similar theory has been proposed, namely an association between periodontal disease and adverse pregnancy outcomes.

Improvements in epidemiology, biostatistics, and molecular biology in the last three decades and the concern among dental researchers to assess the effects of the mouth on general health led to the rehabilitation of the "focal infection theory", with considerable improvements in research methodology as the main factors in this "rebirth". The methodological improvements include a more rational analysis of the biological plausibility, inferences concerning causality, valid interpretation of statistical analyses, and control for bias and confounding and interactional variables. Scientific studies on the relationship between dental infections and chronic and multi-factorial diseases can thus be improved. Periodontal medicine is a new branch of periodontology involving the study of periodontal disease as a possible risk factor for various other diseases, 
including coronary heart disease, diabetes, and adverse pregnancy outcomes.

Studies linking periodontal disease to adverse pregnancy outcomes began in 1996, when Offenbacher et al. 2 claimed to find a strong correlation. Their findings aroused interest mainly because of the impressive odds ratio of 7.9 for pregnant women with periodontal disease and preterm and low birth weight infants. Since then several studies and reviews have been conducted on the relationship between periodontal disease and adverse pregnancy outcomes, but with different methodological designs, some involving serious shortcomings. For example, confounding variables have not been routinely analyzed. Neither is there a balanced view towards the possible relationship between periodontal disease and adverse pregnancy outcomes. The current study aims to provide a critical review of analytical studies regarding periodontal disease as a possible risk factor for adverse pregnancy outcomes.

\section{Methods}

The methods applied in this systematic review cover the literature search strategy and inclusion criteria.

\section{Literature search strategy}

We searched the PubMed, SciELO, and LILACS bibliographic databases and the CAPES thesis/ dissertation database. Standardized methodological filters were used to identify analytical studies and reviews included the following keywords: ((low birth weight OR pre term OR preterm OR prematur* OR immatur*) OR (labor OR pregnancy OR birth OR neonatal OR fetal OR intrauterin*) AND (complication* OR disease* OR adverse)) OR PLBW) AND (periodont*). We also searched reference lists of identified articles and abstracts. The search was limited to studies on human beings written in English or Portuguese. Studies published before December 21, 2005, were included after identification.

\section{Inclusion criteria}

Studies were considered for inclusion if they addressed different clinical, microbiological, or immunological aspects and measurements of destructive periodontal disease and adverse pregnancy outcomes. Analytical studies had to include an estimate of the effect of periodontal disease on pregnancy outcomes and/or statistical tests for comparison of groups. There was a clear heterogeneity among studies concerning measurement of periodontal disease and type of adverse pregnancy outcome used as the dependent variable, so no meta-analysis was performed.

\section{Exclusion criteria}

Cross-sectional studies reporting periodontal conditions in postpartum women, case reports, ecological studies, experimental animal studies, and previous reviews on this subject were excluded.

\section{Results}

Of the 964 papers identified, 36 analytical studies met the inclusion criteria. One of 36 studies 3 was excluded from this analytical review, because it was a duplicate of the study by Dasanayake et al 4 . One cohort data set was analyzed twice in the present review because the study compared both the incidence of preterm birth in treated and untreated women with periodontal disease (clinical trial design) and the proportion of periodontal disease between women with preterm and non-preterm birth (nested case-control in a cohort study analysis) 5 . Therefore, that cohort was included as two studies. Overall, 36 studies were considered in the present systematic review. Twenty-six showed positive associations between periodontal disease and adverse pregnancy outcomes. Figure 1 shows the epidemiological studies included and those showing positive association, by type of epidemiological design.

The main characteristics of the methodology applied in the analytical studies are described according to the study design in Table 1 (casecontrol studies), Table 2 (cohort studies), and Table 3 (clinical trials). There was a clear heterogeneity in the methodology and sample sizes in analytical studies, which may have affected the power and precision in some. A wide range of clinical parameters and indices to assess periodontal disease were used.

The results are presented according to type of study. Overall relevant findings on the target outcome, periodontal disease measurement, and confounding and statistical issues are also presented.

\section{Case control studies}

Findings from case-control studies were analyzed separately according to the target outcome (Table 1). They are: 
Numbers of studies and number showing positive associations between periodontal disease and adverse pregnancy outcomes, by type of epidemiological study design*.

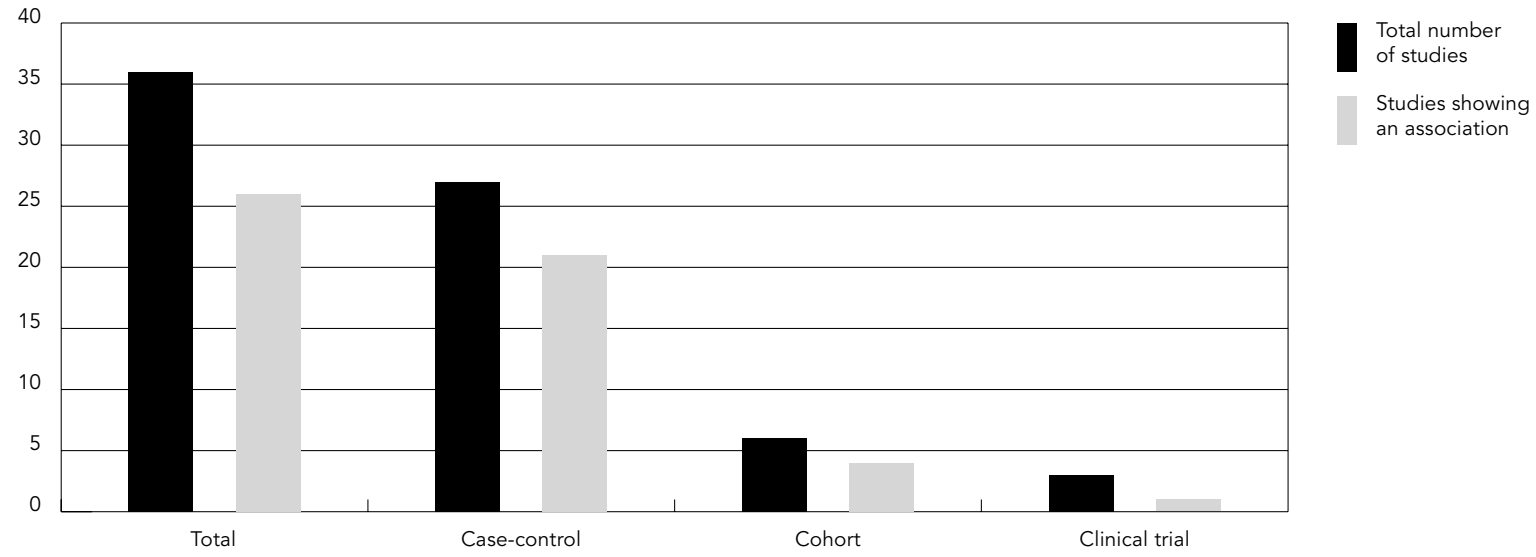

* One article was included twice because it presented results from a clinical trial and a nested case-control analysis in the same cohort study.

\section{- Low birth weight babies}

Low birth weight was the outcome in 8 casecontrol studies 4,6,7,8,9,10,11,12,13. Contradictory findings were reported in 2 studies that used the Community Periodontal Index for Treatment Needs (CPITN) to assess periodontal disease 8,10 . Similar differences were observed in the results between 2 studies using serum IgG levels for periodontal pathogenic species 4,10 , and between 2 studies using clinical attachment level to define periodontal disease 10,13. While the small sample size may explain the lack of power to detect association in the study by Louro 8 , this may not be the case in the other studies not finding an association between periodontal disease and low birth weight 9,10,11,12 .

\section{- Preterm birth}

Nine studies used preterm birth as the target outcome 9,10,11,12,14,15,16,17,18. A strong relationship between Red and Orange microbial complex organisms in periodontal pockets and preterm birth was reported 14,15 . However, no evidence was found to support the systemic dissemination of periodontal pathogens and their products throughout the body, as evidenced by the lack of difference in maternal serum IgG levels and umbilical cord IgG for maternal periodontal pathogens 14 . Other studies differed in their findings. Jarjoura et al. 10 found a significant association between mean periodontal attachment loss and higher prevalence of periodontitis with preterm deliveries, while in a large sample of women $(n=3,738)$, Moore et al. 9 found similar levels of periodontal disease between cases and controls. The absence of an association between periodontal disease and preterm birth was also reported in other studies 11,12,17,18.

\section{- Preterm low birth weight babies}

Preterm low birth weight is the term used to combine the two previous criteria. However, the criteria for preterm low birth weight were not the same in all the studies reviewed.

\section{- Preterm and low birth weight babies}

Eight case control studies considered preterm low birth weight when the newborn was preterm in addition to having low birth weight 2,19,20,21, $22,23,24,25$. Davenport et al. 20 found no difference in CPITN between cases and controls. Similarly, Offenbacher et al. 19, Noack et al. 24, and Budunelli et al. 25 found similar periodontal disease levels between cases and non-cases. Noack et al. 24 also reported no difference in periodontal pathogens between groups.

However, crevicular levels of interleukins and periodontopathogens were higher in women 
Table 1

Characteristics of case-control studies in the relationship between periodontal disease and adverse pregnancy outcomes.

\begin{tabular}{|c|c|c|c|c|c|c|c|c|}
\hline Outcome & $\begin{array}{l}\text { Parameter for } \\
\text { periodontal } \\
\text { disease }\end{array}$ & Reference & Year & $\begin{array}{l}\mathrm{PB} \text { and } \mathrm{BW} \\
\text { assessment }\end{array}$ & $\begin{array}{l}\text { Sample } \\
\text { size }\end{array}$ & Mean age & Variables controlled & OR/SD/NSD \\
\hline \multirow[t]{5}{*}{ LBW } & CPITN & Dasanayake 6 & 1998 & BW: NP & 110 & $27.2 \pm 5.8$ & $\begin{array}{l}\text { Age, diabetes, asthma, } \\
\text { cardiac diseases, } \\
\text { smoking, alcohol, } \\
\text { caffeine, SES, GA, } \\
\text { ethnicity }\end{array}$ & $4.1(1.3-12.8)^{\star}, \star \star$ \\
\hline & CPITN & Sembene et al. 7 & 2000 & BW: NP & 113 & $\begin{array}{l}68 \%<30 \\
(15-39)\end{array}$ & $\begin{array}{l}\text { Age, GUI, previous } \\
\text { abortion }\end{array}$ & $\begin{array}{l}\text { NSD } \\
26.9 \% \\
\text { associated with BW }\end{array}$ \\
\hline & ESI & Louro et al. 8 & 2001 & BW: NP & 26 & $21.0(14-36)$ & $\begin{array}{l}\text { Age, diabetes, } \\
\text { hypertension, GUI, race, } \\
\text { smoking, alcohol, PMH, } \\
\text { PC, SES, ethnicity }\end{array}$ & $\begin{array}{l}\text { NSD for PD Extension } \\
\text { Index }{ }^{\star \star \star} 7.2 \\
(0.4-125.4)^{\star} \text { for PD } \\
\text { severity Index }\end{array}$ \\
\hline & $\begin{array}{l}\text { Specific lgG } \\
\text { serum levels } \\
\text { for periodontal } \\
\text { pathogens }\end{array}$ & $\begin{array}{l}\text { Dasanayake } \\
\text { et al. } 4\end{array}$ & 2001 & BW: NP & 448 & $21.7 \pm 5.4$ & $\begin{array}{l}\text { Age, race, drugs, } \\
\text { smoking and alcohol, } \\
\text { ethnicity }\end{array}$ & $\begin{array}{l}1.02(1.01-1.04)^{\star} \text { for } \\
\text { P. gingivalis IgG } \\
1.15(0.96-1.38)^{\star} \text { for } \\
\text { T. forsythia lgG } \\
0.99(0.95-1.02)^{\star} \text { for } \\
\text { T. denticola lgG }\end{array}$ \\
\hline & $\begin{array}{l}\geq 4 \text { teeth with } \\
A L \geq 4 \mathrm{~mm}\end{array}$ & Cruz et al. 13 & 2005 & BW: NP & 306 & $44 \%<20$ & $\begin{array}{l}\text { Age, parity, previous } \\
\text { periodontal treatment, } \\
\text { alcohol, smoking, } \\
\text { hypertension, diabetes, } \\
\text { MS, SES, GUI }\end{array}$ & $\begin{array}{l}2.2(1.3-3.5)^{\star},{ }^{\star \star} \\
4.0(1.6-11.1)^{\star},{ }^{\star \star} \text { for } \\
\text { schooling } \leq 4 \text { years }\end{array}$ \\
\hline \multirow[t]{4}{*}{ PB } & $\begin{array}{l}\text { Periodontal } \\
\text { pathogens } \\
\text { quantification, } \\
\text { maternal lgG } \\
\text { serum levels and } \\
\text { umbilical cord } \\
\text { IgM for } \\
\text { periodontal } \\
\text { pathogens }\end{array}$ & $\begin{array}{l}\text { Madianos } \\
\text { et al. } 14\end{array}$ & 2001 & PB: NP & 812 & $26.7 \pm 6.4$ & $\begin{array}{l}\text { Age, GUI, race, } \\
\text { smoking, PMH, PC, } \\
\text { dietary habits, MS }\end{array}$ & $\begin{array}{l}4.3(2.11-8.90)^{\star} \text { for } \\
\text { Orange complex } \\
2.2(1.48-3.79)^{\star} \text { for } \\
\text { Red complex**}\end{array}$ \\
\hline & $\begin{array}{l}\text { CAL, PPD, BOP, } \\
\text { periodontal } \\
\text { pathogens semi- } \\
\text { quantification }\end{array}$ & $\begin{array}{l}\text { Hasegawa } \\
\text { et al. } 15\end{array}$ & 2003 & PB: NP & 88 & $29.7 \pm 4.6$ & $\begin{array}{l}\text { Age, PMH, GUI, } \\
\text { smoking, parity, } \\
\text { antibiotics, BMI }\end{array}$ & $\begin{array}{l}\text { SD for PPD mean, } \\
\% C A L \geq 3 \mathrm{~mm} \\
\text { SD for } T \text {. forsythia } \\
\text { total numbers }\end{array}$ \\
\hline & $\begin{array}{l}\mathrm{PI}, \mathrm{BOP}, \mathrm{PPD}, \\
\mathrm{CAL}>5 \% \text { with } \\
\mathrm{PPD} \geq 5 \mathrm{~mm} \text { or } \\
>5 \% \text { sites with } \\
\mathrm{AL} \geq 3 \mathrm{~mm} \mathrm{~m} \mathrm{~N}-\alpha \\
\text { and IL1- } \beta \\
\text { polymorphism }\end{array}$ & Moore et al. 18 & 2004 & PB: NP & 130 & $29.4 \pm 6.4$ & $\begin{array}{l}\text { Age, ethnicity, } \\
\text { parity, hypertension, } \\
\text { diabetes, SES }\end{array}$ & $\begin{array}{l}\text { NSD for any } \\
\text { periodontal } \\
\text { parameters }\end{array}$ \\
\hline & $\begin{array}{l}\text { CAL, PPD, BOP, } \\
\text { PI }\end{array}$ & Moore et al. 17 & 2005 & PB: NP & 154 & $29.4 \pm 6.3$ & $\begin{array}{l}\text { Age, parity, diabetes, } \\
\text { antibiotics, hypertension, } \\
\text { SES, GUI, ethnicity }\end{array}$ & $\begin{array}{l}\% \text { of sites PPD } \geq 5 \mathrm{~mm} \\
\text { was lower in cases }\end{array}$ \\
\hline PB§ & $\begin{array}{l}\mathrm{AL}>5 \mathrm{~mm} \text { in any } \\
\text { one sextant } \\
\text { ESI }\end{array}$ & $\begin{array}{l}\text { Goepfert } \\
\text { et al. } 16\end{array}$ & 2004 & PB: NP & 139 & $23.9 \pm 5.4$ & $\begin{array}{l}\text { Age, ethnicity, } \\
\text { smoking, } \mathrm{PMH}, \mathrm{PC}\end{array}$ & $\begin{array}{l}2.6^{\star}(1.1-6.2)^{\star \star} \\
\text { SD for Extent } 5^{\star \star}\end{array}$ \\
\hline $\begin{array}{l}\text { LBW } \\
\text { PB }\end{array}$ & $\begin{array}{l}\geq 5 \text { sites with } \\
C A L \geq 3 \mathrm{~mm}, \\
\text { periodontal } \\
\text { pathogens } \\
\text { quantification, } \\
\text { serum IgG levels } \\
\text { against periodontal } \\
\text { species }\end{array}$ & $\begin{array}{l}\text { Jarjoura et al. } 10 \\
\text { | }\end{array}$ & 2004 & $\begin{array}{l}\text { PB: UE } \\
\text { (before } \\
\text { 20th week } \\
\text { BW: NP }\end{array}$ & 203 & $28.6 \pm 6.7$ & $\begin{array}{l}\text { Age, GUI, race, smoking, } \\
\text { BMI, PMH, SES }\end{array}$ & $\begin{array}{l}\text { PB: } 2.75(1.01-7.54)^{\star} \\
\text { for } A L>3 \mathrm{~mm}^{\star \star} \\
\text { LBW: } 1.99(0.73-5.45)^{\star} \\
\text { for } A L>3 \mathrm{~mm}^{\star \star \star} \\
\text { NSD for periodontal } \\
\text { pathogen } \\
\text { quantification, serum } \\
\text { lgG levels against } \\
\text { periodontal species }\end{array}$ \\
\hline
\end{tabular}

(continues) 
Table 1 (continued)

\begin{tabular}{|c|c|c|c|c|c|c|c|c|}
\hline Outcome & $\begin{array}{l}\text { Parameter for } \\
\text { periodontal } \\
\text { disease }\end{array}$ & Reference & Year & $\begin{array}{l}\text { PB and BW } \\
\text { assessment }\end{array}$ & $\begin{array}{l}\text { Sample } \\
\text { size }\end{array}$ & Mean age & Variables controlled & OR/SD/NSD \\
\hline & $\begin{array}{l}\text { Mean PPD } \\
\text { and CAL } \\
\% P P D \geq 4 \text { and } \\
\geq 5 \mathrm{~mm} \\
\% A L \geq 2 \text { and } \\
3 \mathrm{~mm}\end{array}$ & Moore et al. 9 & 2004 & $\begin{array}{l}\text { PB: UE } \\
\text { (at } 12^{\text {th }} \text { week) } \\
\text { BW: NP }\end{array}$ & 3,738 & $29.9 \pm 5.5$ & $\begin{array}{l}\text { Age, GUI, race, } \\
\text { smoking, alcohol, } \mathrm{PMH} \text {, } \\
\text { antibiotics, SES }\end{array}$ & $\begin{array}{l}\text { NSD for } \mathrm{LBW}^{\star \star \star \star} \\
\text { NSD for } \mathrm{PB}^{\star \star \star}\end{array}$ \\
\hline & $\begin{array}{l}\% \mathrm{BOP}, \% \mathrm{PI} \\
\mathrm{PPD} \text { mean and } \\
\% \mathrm{PPD}>3 \mathrm{~mm}\end{array}$ & Moreu et al. 12 & 2005 & $\begin{array}{l}\text { PB: NP } \\
\text { BW: NP }\end{array}$ & 96 & $\begin{array}{l}29.32 \\
(18-40)\end{array}$ & $\begin{array}{l}\text { Age, smoking, alcohol, } \\
\text { drugs, parity, gestational } \\
\text { weeks }\end{array}$ & $\begin{array}{l}\text { PB: } 0.99 *(\% \text { PPD } \\
\left.>3 m^{\star \star \star}\right) ; 0.87^{\star} \\
\left(P P D \text { mean }{ }^{\star \star \star}\right) \\
\text { LBW: } 1.99^{\star}(\% \text { PPD } \\
\left.>3 m^{\star *}\right) ; 1.04^{\star} \\
\left.\text { (PPD mean }{ }^{\star \star \star}\right)\end{array}$ \\
\hline $\begin{array}{l}\text { LBW } \\
\text { PB } \\
\text { LBW } \\
\text { or PB }\end{array}$ & $\begin{array}{l}\geq 1 \text { sites with } \\
\text { PPD } \geq 3.5 \mathrm{~mm} \\
\geq 4 \text { sites with } \\
P P D \geq 3.5 \mathrm{~mm}\end{array}$ & $\begin{array}{l}\text { Lunardelli \& } \\
\text { Peres } 11\end{array}$ & 2005 & $\begin{array}{l}\text { PB: NP } \\
\text { BW: NP }\end{array}$ & 449 & $\begin{array}{l}91.3 \%>19 \\
8.7 \% \leq 19\end{array}$ & $\begin{array}{l}\text { Age, diabetes, cardiac } \\
\text { disease, parity, race, SES, } \\
\text { PMH, GUI, PC, drugs, } \\
\text { smoking, BMI, ethnicity }\end{array}$ & $\begin{array}{l}2.7(0.7-9.7)^{\star} \text { for } \mathrm{PB}^{\star \star \star} \\
2.0(0.8-4.8)^{\star} \\
\text { for } \mathrm{LBW}^{\star \star \star} \\
1.5(0.5-4.4)^{\star} \\
\text { for PTLBW }\end{array}$ \\
\hline \multirow[t]{5}{*}{$\begin{array}{l}\text { LBW } \\
\text { or PB }\end{array}$} & ESI & Cardoso 26 & 1999 & $\begin{array}{l}\text { PB: NP } \\
\text { BW: NP }\end{array}$ & 287 & $27.3 \pm 4.1$ & $\begin{array}{l}\text { Age, diabetes, } \\
\text { hypertension, GUI, race, } \\
\text { smoking, alcohol, PMH, } \\
\text { PC, SES }\end{array}$ & NSD for $E S I^{\star \star \star}$ \\
\hline & $\begin{array}{l}\text { Mean PPD and } \\
\text { PI, \% of sites } \\
\text { with BOP, with } \\
\text { calculus and } \\
\text { periodontal } \\
\text { pathogens semi- } \\
\text { quantification }\end{array}$ & $\begin{array}{l}\text { Mitchell-Lewis } \\
\text { et al. } 5\end{array}$ & 2001 & $\begin{array}{l}\text { PB: NP } \\
\text { BW: NP }\end{array}$ & 164 & $16.7 \pm 1.4$ & $\begin{array}{l}\text { Age, Diabetes, GUI, race, } \\
\text { drugs, smoking, alcohol, } \\
\text { PMH, PC, SES }\end{array}$ & $\begin{array}{l}\text { NSD for clinical } \\
\text { parameters } \\
\text { SD for P. nigrescens; } \\
\text { T. forsythensis, } \\
\text { Camplobacter rectus, } \\
\text { E. corrodens and } \\
\text { E. nodatum }{ }^{\star \star}\end{array}$ \\
\hline & $\begin{array}{l}\text { Mean PPD, BOP, } \\
\text { calculus, CPITN }\end{array}$ & Mokeen et al. 27 & 2004 & $\begin{array}{l}\text { PB: NP } \\
\text { BW: NP }\end{array}$ & 90 & $29.3 \pm 6.6$ & $\begin{array}{l}\text { Age, diabetes, } \\
\text { hypertension, GUI, } \\
\text { smoking, PMH, } \\
\text { antibiotics, parity, PC, } \\
\text { Previous periodontal } \\
\text { treatment, SES }\end{array}$ & $\begin{array}{l}4.21(1.99-8.93)^{\star} \text { for } \\
\text { mean CPITN } \\
\text { SD for mean PPD, } \\
\text { mean BOP, mean } \\
\text { calculus, mean } \\
\text { CPITN** }\end{array}$ \\
\hline & $\begin{array}{l}\geq 1 \text { site with PPD } \\
\geq 4 \mathrm{~mm} \text { and } \\
\geq 50 \% \text { BOP }\end{array}$ & Radnai et al. 29 & 2004 & $\begin{array}{l}\text { PB: NP } \\
\text { BW: NP } \\
\text { PROM: NP } \\
\text { TPL }\end{array}$ & 85 & 27.9 & $\begin{array}{l}\text { Age, diabetes, } \\
\text { hypertension, parity, } \\
\text { MS, PC, SES, smoking, } \\
\text { alcohol, drugs }\end{array}$ & $5.46(1.72-17.32)^{\star},{ }^{* \star}$ \\
\hline & $\begin{array}{l}\geq 1 \text { site with PPD } \\
\geq 5 \mathrm{~mm} \text { in each } \\
\text { quadrant and } \\
\text { Red and Orange } \\
\text { clusters }\end{array}$ & $\begin{array}{l}\text { Dörtbudak } \\
\text { et al. } 28\end{array}$ & 2005 & $\begin{array}{l}\text { PB: NP } \\
\text { BW: NP }\end{array}$ & 36 & $31.1 \pm 3.4$ & $\begin{array}{l}\text { Age, PC, smoking, } \\
\text { diabetes, alcohol, BMI }\end{array}$ & $\begin{array}{l}\text { SD for } \% \text { periodontitis } \\
\text { between groups }{ }^{\star \star} \\
\text { SD for } \% \text { of Orange } \\
\text { and Red clusters }{ }^{\star \star}\end{array}$ \\
\hline \multirow[t]{4}{*}{$\begin{array}{l}\text { LBW } \\
\text { and PB }\end{array}$} & $\begin{array}{l}\text { ESI with a } 4 \mathrm{~mm} \\
\mathrm{AL} \text { threshold } \\
\text { value, PGE-2 and } \\
\text { IL-1 } \beta \text { GCF mean } \\
\text { and periodontal } \\
\text { pathogens } \\
\text { quantification }\end{array}$ & $\begin{array}{l}\text { Offenbacher } \\
\text { et al. } 19\end{array}$ & 1998 & $\begin{array}{l}\text { PB: NP } \\
\text { BW: NP }\end{array}$ & 44 & NP & $\begin{array}{l}\text { Age, PMH, smoking, } \\
\text { alcohol, GUI }\end{array}$ & $\begin{array}{l}\text { SD for PGE-2 and } \\
\text { IL-1 } \beta \text { crevicular levels } \\
\text { SD for Red complex**} \\
\text { NSD for PD Extension } \\
\text { Index DP*** }\end{array}$ \\
\hline & CPITN & Davenport 20 & 2002 & $\begin{array}{l}\text { PB: NP } \\
\text { BW: NP }\end{array}$ & 743 & $\begin{array}{l}26.8 \\
(16->35)\end{array}$ & $\begin{array}{l}\text { Age, hypertension, GUI, } \\
\text { race, smoking, alcohol, } \\
\text { PMH, PC, dietary habits, } \\
\text { SES }\end{array}$ & 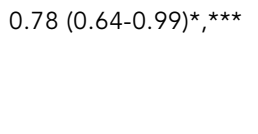 \\
\hline & $\begin{array}{l}\geq 4 \text { sites with } C A L \\
\text { and } P P D \geq 3 \mathrm{~mm}\end{array}$ & $\begin{array}{l}\text { Moliterno } \\
\text { et al. } 21\end{array}$ & 2005 & $\begin{array}{l}\text { PB: Capurro } \\
\text { score } \\
\text { BW: digital } \\
\text { scale }\end{array}$ & 151 & $25.0 \pm 6.4$ & $\begin{array}{l}\text { Age, diabetes, } \\
\text { hypertension, GUI, race, } \\
\text { drugs, smoking, alcohol, } \\
\text { PMH, PC, SES, ethnicity }\end{array}$ & $3.48(1.17-10.36)^{\star},{ }^{\star \star}$ \\
\hline & ESI & $\begin{array}{l}\text { Offenbacher } \\
\text { et al. } 2\end{array}$ & 1996 & $\begin{array}{l}\text { PB: UE (at 24th } \\
\text { week) and } \\
\text { Dubowitz } \\
\text { examination } \\
\text { BW: NP }\end{array}$ & 124 & $\begin{array}{l}23.5 \\
(14-40)\end{array}$ & $\begin{array}{l}\text { Age, diabetes, } \\
\text { hypertension, GUI, race, } \\
\text { drugs, smoking, alcohol, } \\
\text { PMH, PC, parity }\end{array}$ & $\begin{array}{l}\text { Primiparous: } 7.9 \\
(1.50-41.1)^{\star},{ }^{\star \star} \\
\text { Non primiparous: } 7.5 \\
(1.95-28.8)^{\star},{ }^{\star \star}\end{array}$ \\
\hline
\end{tabular}


Table 1 (continued)

\begin{tabular}{|c|c|c|c|c|c|c|c|c|}
\hline Outcome & $\begin{array}{l}\text { Parameter for } \\
\text { periodontal } \\
\text { disease }\end{array}$ & Reference & Year & $\begin{array}{l}\text { PB and BW } \\
\text { assessment }\end{array}$ & $\begin{array}{l}\text { Sample } \\
\text { size }\end{array}$ & Mean age & Variables controlled & OR/SD/NSD \\
\hline & $\begin{array}{l}\text { PDI, PGE- } 2 \text { and } \\
\text { IL-1 } \beta \text { levels in } \\
\text { serum and GCF }\end{array}$ & $\begin{array}{l}\text { Konopka } \\
\text { et al. } 24\end{array}$ & 2003 & $\begin{array}{l}\text { PB: NP } \\
\text { BW: NP }\end{array}$ & 84 & $\begin{array}{l}27.5 \\
(16-41)\end{array}$ & $\begin{array}{l}\text { Age, parity, antibiotics, } \\
\text { PMH, GUI, smoking }\end{array}$ & $\begin{array}{l}\text { Primiparous: } 3.90 \\
(0.93-19.14) \text { for } \\
\text { PDI* }^{* *} \text { and SD for } \\
\text { PGE-2 and IL1 } \beta \text { GCF } \\
\text { levels and for serum } \\
\text { PGE-2** } \\
\text { Non primiparous: } \\
1.26(0.53-3.06) \text { for } \\
\text { PDI*,** and DS for } \\
\text { PGE-2 and IL-1 } \beta \\
\text { GCF levels** }\end{array}$ \\
\hline & $\begin{array}{l}\text { CPITN, PGE-2 } \\
\text { and IL-1 } \beta \\
\text { crevicular levels }\end{array}$ & Carta et al. 23 & 2004 & $\begin{array}{l}\text { PB: NP } \\
\text { BW: NP }\end{array}$ & 92 & NP & $\begin{array}{l}\text { Age, diabetes, } \\
\text { hypertension, } \mathrm{PMH} \text {, } \\
\text { smoking, alcohol, } \\
\text { GUI, PC }\end{array}$ & $\begin{array}{l}\text { CPITN }=4: \text { controls }= \\
4.3 \%, \text { cases }=40 \% \\
\text { SD for PGE-2 and } \\
\text { IL- } 1 \beta \text { ** }\end{array}$ \\
\hline & $\begin{array}{l}\text { PPD, BOP and PI } \\
\text { mean, \% of sites } \\
\text { with BOP and } \\
\text { plaque and } \\
\text { periodontal } \\
\text { pathogens semi- } \\
\text { quantification }\end{array}$ & $\begin{array}{l}\text { Buduneli } \\
\text { et al. } 25\end{array}$ & 2005 & $\begin{array}{l}\text { PB: LMP } \\
\text { BW: NP }\end{array}$ & 181 & $\begin{array}{l}24.9 \pm 4.9 \\
(18-35)\end{array}$ & $\begin{array}{l}\text { Age, diabetes, } \\
\text { hypertension, parity, } \\
\text { smoking, GUI, Previous } \\
\text { periodontal treatment, } \\
\text { SES }\end{array}$ & $\begin{array}{l}\text { NSD for periodontal } \\
\text { clinical parameters } \\
0.35^{\star} \text { for } \\
\text { P. nigrescens } \\
0.18^{\star \star} \text { for } A \text {. actino- } \\
\text { mycetemcomitans } \\
3.82^{\star} \text { for } P \text {. micros } \\
7.15^{\star} \text { for } C \text {. rectus } \\
\text { (* }\end{array}$ \\
\hline & $\begin{array}{l}\mathrm{PPD}, \mathrm{AL}, \% \text { sites } \\
\mathrm{AL} \geq 3 \mathrm{~mm}, \mathrm{BOP}, \\
\mathrm{IP} \text { mean, IL-1 } \beta \\
\text { crevicular and } \\
\text { periodontal } \\
\text { pathogens semi- } \\
\text { quantification }\end{array}$ & Noack et al. 26 & 2005 & $\begin{array}{l}\text { PB: NP } \\
\text { BW: NP }\end{array}$ & 59 & $27.8-30.3$ & $\begin{array}{l}\text { Age, BMI, GUI, diabetes, } \\
\text { parity, smoking, drugs, } \\
\text { alcohol, PMH, stress, } \\
\text { SES, PC, antibiotic }\end{array}$ & $\begin{array}{l}\text { NSD for any } \\
\text { periodontal } \\
\text { parameters }{ }^{\star \star \star} \\
0.73(0.13-4.19)^{\star},{ }^{\star \star \star}\end{array}$ \\
\hline
\end{tabular}

* OR $=$ Odds ratio.

** SD = Significant difference between groups $(p \leq 0.05)$

$\star \star \star N S D=$ No statistical difference between groups.

LBW $=$ Low birth weight; $\mathrm{PB}=$ Preterm birth $(<37$ weeks gestational age $)$; PB§ = Preterm birth $(<32$ weeks of gestation);

$\mathrm{PROM}=$ Premature rupture of membranes; TPL = Threatened preterm labor; CPITN = Community Periodontal Index

of Treatment Needs; ESI = Extension and Severity Index; Ig = Immunoglobulin; PPD = Periodontal pocket depth;

$\mathrm{CAL}=$ Clinical attachment level; $\mathrm{AL}=$ Attachment loss; $\mathrm{BOP}=$ Bleeding on probing; $\mathrm{PI}=\mathrm{Plaque}$ index:

$\mathrm{PGE}_{-2}=$ prostaglandin $\mathrm{E}_{-2} ; \mathrm{IL}-1 \beta=$ interleukin $-1 \beta ; \mathrm{GCF}=$ Gingival crevicular fluid; PDI = Periodontal Disease Index;

$\mathrm{UE}=$ Ultrasound examination; NP = Data not presented; SES = Socioeconomic status; GA = Gestational age;

$\mathrm{GUI}=$ Genito-urinary infection; $\mathrm{PMH}=$ Pregnancy medical history; $\mathrm{PC}=$ Prenatal care; $\mathrm{MS}=\mathrm{Marital}$ status; $\mathrm{BMI}=\mathrm{Body} \mathrm{Mass}$ Index.

with preterm and low birth weight babies 19 . Four other case-control studies reported an increased risk in the periodontal disease group, although using different definitions for periodontal disease 2,21,22,23. Carta et al. 23 also found differences for prostaglandin E-2 (PGE-2) and interlukin- $1 \beta$ (IL-1 $\beta$ ) crevicular levels between groups with and without preterm and low birth weight.

\section{- Preterm or low birth weight babies}

In five studies, outcome was defined as preterm or low birth weight 5,11,26,27,28. Mokeen et al. 27 and Dörtbudak et al. 28 reported different levels of periodontal disease between cases and controls, whereas other authors found no differences in periodontal status between groups
$5,11,26$. In another study, postpartum women with clinical periodontitis had an increased risk of low birth weight, preterm birth, premature rupture of membranes, or threatened preterm labor 29 .

Four studies reported differences in periodontal pathogen levels in mothers of preterm and non-preterm low birth weight babies 5,19, 25,28 , although 3 studies showed no difference in periodontal disease status. The wide variety of methods for periodontal disease assessment, the possible presence of confounders, and different methods for assessing outcome may explain the disagreement among case-control studies. 
Characteristics of cohort studies in the relationship between periodontal disease and adverse pregnancy outcomes.

\begin{tabular}{|c|c|c|c|c|c|c|c|c|}
\hline Outcome & $\begin{array}{l}\text { Parameter for } \\
\text { periodontal } \\
\text { disease }\end{array}$ & Reference & Year & $\begin{array}{l}\mathrm{PB} \text { and } \mathrm{BW} \\
\text { assessment }\end{array}$ & $\begin{array}{l}\text { Sample } \\
\text { size }\end{array}$ & Mean age & Variables controlled & OR/RR/SD/NSD \\
\hline PB & $\begin{array}{l}\mathrm{PPD} \geq 4 \mathrm{~mm} \text { in } \\
\text { Ramfjord teeth }\end{array}$ & $\begin{array}{l}\text { Holbrook } \\
\text { et al. } 33\end{array}$ & 2004 & $\begin{array}{l}\text { PB: UE (at } \\
\text { 18th-19th week) }\end{array}$ & 96 & NP & $\begin{array}{l}\text { Age, parity, race, GUI, } \\
\text { smoking, antibiotics, } \\
\text { PMH }\end{array}$ & $\begin{array}{l}\text { NSD for } \geq 4 \text { PPD } \\
\geq 4 \mathrm{~mm}^{\star \star \star}\end{array}$ \\
\hline $\begin{array}{l}\text { LBW } \\
\text { PB }\end{array}$ & $\begin{array}{l}\text { Gingivitis: } \mathrm{BOP} \\
>5 \% \text { without } \geq 2 \\
\text { sites with } \mathrm{CAL} \\
>6 \mathrm{~mm} \text { and }<2 \\
\text { sites with } \mathrm{PPD} \\
\geq 5 \mathrm{~mm} \\
\text { Periodontitis: } \geq 1 \\
\text { site with } \mathrm{PPD} \\
\geq 5 \mathrm{~mm} \text { and } \geq 2 \\
\text { sites with } \mathrm{CAL} \\
>6 \mathrm{~mm} \text { and } \mathrm{BOP} \\
>5 \%\end{array}$ & Marin et al. 34 & 2005 & PB: NP & 152 & $23.3 \pm 5.7$ & $\begin{array}{l}\text { Age, smoking, diabetes, } \\
\text { alcohol, SES, race, } \\
\text { Previous periodontal } \\
\text { treatment }\end{array}$ & $\begin{array}{l}\text { SD for infant birth } \\
\text { weight between } \\
\text { healthy and } \\
\text { periodontitis for } \\
\text { women }>25 \text { years } \\
\text { old**} \\
\text { NSD for } \% \text { of preterm } \\
\text { among groups }{ }^{\star \star \star}\end{array}$ \\
\hline $\begin{array}{l}\text { LBW } \\
\text { or PB }\end{array}$ & $\begin{array}{l}\geq 4 \text { teeth with } 1 \\
\text { or more sites with } \\
\mathrm{PPD}=4 \mathrm{~mm} \text { and } \\
\mathrm{AL}=3 \mathrm{~mm} \text { in the } \\
\text { same site }\end{array}$ & López et al. 30 & 2002 & $\begin{array}{l}\text { PB: UE (NP), } \\
\text { LMP, SPE/PNE, } \\
\text { Ballard } \\
\text { neonatal } \\
\text { assessment }\end{array}$ & 639 & $25.0 \pm 4.5$ & $\begin{array}{l}\text { Age, GUI, smoking, } \\
\text { PMH,PC, MS, SES }\end{array}$ & $3.5(1.7-7.3)^{\star \star}, \#$ \\
\hline \multirow[t]{2}{*}{$\begin{array}{l}\text { LBW } \\
\text { and PB }\end{array}$} & $\begin{array}{l}\text { Moderate to } \\
\text { severe: } \geq 4 \text { sites } \\
\text { with at least } \\
P P D=5 \mathrm{~mm} \text { and } \\
A L=2 \mathrm{~mm} \\
\text { Moderate: } 1 \text { to } \\
4 \text { sites with } \\
P P D>3 \mathrm{~mm} \text { and } \\
A L>2 \mathrm{~mm} \\
\text { Progression/ } \\
\text { Incidence: } \geq 4 \text { sites } \\
\text { with increasing } \\
\text { PPD of } \geq 2 \mathrm{~mm} \text {. }\end{array}$ & $\begin{array}{l}\text { Offenbacher } \\
\text { et al. } 32\end{array}$ & 2001 & $\begin{array}{l}\text { PB: UE (before } \\
\text { 20th week) } \\
\text { pelvic exam } \\
\text { BW: recorded } \\
\text { at delivery or } \\
\text { in the neonatal } \\
\text { intensive care }\end{array}$ & 812 & $26.7 \pm 6.4$ & $\begin{array}{l}\text { Age, GUI, race, } \\
\text { smoking, PMH, PC, } \\
\text { dietary habits, MS }\end{array}$ & $\begin{array}{l}\text { PD moderate to } \\
\text { severe: } 2.23^{\star *}, \# \\
\text { PD moderate: } 1^{\star \star *}, \# \\
\text { PD progression } \\
\text { DP: } 1.4^{\star *}, \#\end{array}$ \\
\hline & $\begin{array}{l}\text { Mean PPD, PI } \\
\text { and BOP greater } \\
\text { than the median }\end{array}$ & $\begin{array}{l}\text { Rajapakse } \\
\text { et al. } 35\end{array}$ & 2005 & PB: UE (NP) & 227 & $24.2 \pm 4.2$ & $\begin{array}{l}\text { Age, smoking, diabetes, } \\
\text { alcohol, SES, race, } \\
\text { hypertension, Previous } \\
\text { periodontal treatment, PC }\end{array}$ & $1.9(0.7-5.4)^{\star},{ }^{\star \star \star}$ \\
\hline
\end{tabular}

* OR $=$ Odds ratio.

** SD = Significant difference between groups $(p \leq 0.05)$

$\star \star \star N S D=$ No statistical difference between groups $(p>0.05)$.

$\#$ RR = Relative risk.

LBW = Low birth weight; $\mathrm{PB}=$ Preterm birth; $\mathrm{AL}=$ Attachment loss; $\mathrm{PPD}=$ Periodontal pocket depth; $\mathrm{BOP}=\mathrm{Bleeding}$ on probing

$N P=$ Data not presented; $U E$ = Ultrasound examination; SPE/PNE = Sequential physical examinations and post-natal examination;

LMP = Last menstrual period; $\mathrm{PMH}=$ Pregnancy medical history; $\mathrm{PC}=$ Prenatal care; $\mathrm{GUI}=$ Genito-urinary infection;

MS = Marital status; SES = Socioeconomic status; SRP = Scaling and root planing. 
Table 3

Characteristics of clinical trial studies in the relationship between periodontal disease and adverse pregnancy outcomes.

\begin{tabular}{|c|c|c|c|c|c|c|c|c|c|}
\hline Outcome & $\begin{array}{l}\text { Parameter } \\
\text { for PD }\end{array}$ & Reference & Year & $\begin{array}{l}\text { PB and BW } \\
\text { assessment }\end{array}$ & $\begin{array}{l}\text { Sample } \\
\text { size }\end{array}$ & Mean age & Variables controlled & Intervention & OR/SD/NSD \\
\hline $\begin{array}{l}\text { PB } \\
\text { LBW } \\
\text { LBW } \\
\text { or PB }\end{array}$ & $\begin{array}{l}\geq 4 \text { teeth with } \\
1 \text { or more sites } \\
\text { with } P P D= \\
4 \mathrm{~mm} \text { and } \\
\mathrm{AL}=3 \mathrm{~mm} \text { in } \\
\text { the same sites }\end{array}$ & $\begin{array}{l}\text { López } \\
\text { et al. } 36\end{array}$ & 2002 & $\begin{array}{l}\text { PB: UE (9th to } \\
\text { 24th weeks), } \\
\text { LMP, SPE/ } \\
\text { PNE, }\end{array}$ & 400 & $27.5 \pm 4.4$ & $\begin{array}{l}\text { Age, GUI, smoking, } \\
\text { PMH, PC, MS, } \\
\text { antibiotics, SES }\end{array}$ & $\begin{array}{l}\mathrm{SRP}+\text { plaque } \\
\text { control } \\
\text { instruction }\end{array}$ & $\begin{array}{l}\text { PB: } 5.48 \\
(1.17-27.71)^{\star},{ }^{\star \star} \\
\text { LBW: } 6.26 \\
(0.73-53.78)^{\star},{ }^{\star \star} \\
\text { LBW or PB: } 5.49 \\
(1.65-18.22)^{\star},{ }^{\star \star}\end{array}$ \\
\hline $\begin{array}{l}\text { LBW } \\
\text { or PB }\end{array}$ & $\begin{array}{l}\text { PPD and plaque } \\
\text { index mean, } \\
\% \text { of sites with } \\
\text { BOP, with } \\
\text { calculus and } \\
\text { periodontal } \\
\text { pathogens semi- } \\
\text { quantification }\end{array}$ & $\begin{array}{l}\text { Mitchell- } \\
\text { Lewis } \\
\text { et al. } 5\end{array}$ & 2001 & $\begin{array}{l}\text { PB: NP } \\
\text { BW: NP }\end{array}$ & 164 & $16.7 \pm 1.4$ & $\begin{array}{l}\text { Age, diabetes, GUI, } \\
\text { race, drugs, smoking, } \\
\text { alcohol, PMH, PC, SES }\end{array}$ & $\begin{array}{l}\text { SRP + plaque } \\
\text { control } \\
\text { instruction }\end{array}$ & ID $=28.6 \% \ddagger$ \\
\hline PB & $\begin{array}{l}>3 \text { sites with } \\
A L \geq 3 \mathrm{~mm}\end{array}$ & $\begin{array}{l}\text { Jeffcoat } \\
\text { et al. } 37\end{array}$ & 2003 & $\begin{array}{l}\text { PB: UE (NP) } \\
\text { and last } \\
\text { menstrual } \\
\text { period }\end{array}$ & 366 & $22.5 \pm 4.6$ & $\begin{array}{l}\text { Age, GUI, race, MS, } \\
\text { smoking, PMH, } \\
\text { Previous periodontal } \\
\text { treatment, antibiotics, } \\
\text { mouth rinse, body } \\
\text { mass index, }\end{array}$ & $\begin{array}{l}\mathrm{SRP}+\text { placebo } \\
\mathrm{SRP}+ \\
\text { metronidazole }\end{array}$ & $\begin{array}{l}\text { Gestational age } \\
<37 \text { weeks } \\
\text { SRP + placebo: } 0.5 \\
(0.2-1.3)^{\star},{ }^{\star \star \star} \\
\text { SRP }+ \text { metronidazole: } \\
1.4(0.7-2.9)^{\star},{ }^{\star \star \star} \\
\text { Gestational age } \\
<35 \text { weeks } \\
\text { SRP + placebo: } 0.2 \\
(0.02-1.4)^{\star},{ }^{\star \star \star} \\
\text { SRP }+ \text { metronidazole: } \\
0.7(0.2-2.4)^{\star},{ }^{\star \star \star}\end{array}$ \\
\hline
\end{tabular}

${ }^{\star} \mathrm{OR}=$ Odds ratio.

$\star \star$ SD $=$ Significant difference between groups $(p \leq 0.05)$

$\star \star \star N S D=$ No statistical difference between groups $(p>0.05)$

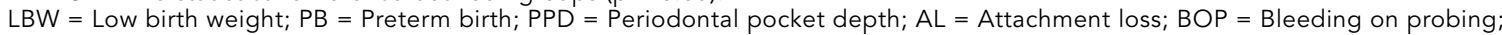

$\mathrm{UE}=$ Ultrasound examination; SPE/PNE = Sequential physical examinations and post-natal examination; NP = Data not presented;

$\mathrm{GUI}=$ Genito-urinary infection; $\mathrm{PMH}=$ Pregnancy medical history; $\mathrm{PC}=$ Prenatal care; $\mathrm{MS}=$ Marital status;

$\mathrm{SES}=$ Socioeconomic status; $\mathrm{SRP}=$ Scaling and root planing; ID = Incidence difference .

\section{Cohort studies}

Six cohort studies were identified $30,31,32,33,34,35$ (Table 2). Preterm birth was the outcome in 3 studies. Jeffcoat et al. 31 found a strong dose-response relationship between periodontal attachment loss and gestational age at birth. The authors used odds ratio rather than relative risk as the measure of association, which may have distorted the association, since preterm birth cannot be considered an uncommon event. Holbrook et al. 33 and Marin et al. 34 found no association between periodontal disease and preterm birth.

López et al. 30 found a 3.5 odds ratio for low birth weight or preterm babies in women with moderate periodontal disease. As in Jeffcoat's cohort study 31 , the use of odds ratios to estimate the association may have produced a bias in the findings. Offenbacher et al. 32 detected the same risk of low birth weight and preterm infants in women with moderate periodontal disease, but the risk was twice as high in women with severe periodontal disease as compared to periodontally healthy women.

Of six cohort studies, only one presented the numbers of women lost to follow-up ( $12 \%$ in the Chilean cohort study) 30 . The proportions of women excluded were $11.5 \%$ and $11.2 \%$ in healthy women and the periodontal disease group respectively. Since losses to follow-up represented $72 \%$ of excluded women, it is likely that the two groups had similar losses to followup 30. As mentioned above, the other cohort studies failed to report losses to follow-up 31 .

\section{Clinical trials}

Three clinical trials were conducted to evaluate the effect of periodontal treatment in reducing 
adverse pregnancy outcomes 5,36,37. Of these, only one showed that periodontal intervention in pregnant women decreased the risk of preterm birth, low birth weight, or preterm birth or low birth weight 36 (Table 3). The risk of preterm birth was not decreased in women submitted to scaling and root planing, or in those with scaling and root planing plus metronidazole 5,37.

Of the 3 clinical trials, only one conducted intention-to-treat analysis 36 , in which the odds ratios were higher than those from the protocol analysis. Random assignment of patients receiving periodontal therapy was described in two studies 36,37 .

Among three clinical trials analyzed, two reported losses to follow-up. Mitchell-Lewis et al. 5 did not present the losses separately for each group. The overall percentage of the recruited sample lost to follow-up was $8 \%$. In the other clinical trial, a total of $12.7 \%$ of women were lost to follow-up (18.5\% in the treatment group and $6 \%$ in the control group) 36 .

Three cohort studies and one clinical trial did not present information on loss to followup $31,32,33,36$. They account for more than $50 \%$ of prospective studies conducted to date. If the proportion of women lost in such studies were large, their validity would be affected.

\section{Outcome measures}

Most of the studies did not present information on how birth weight and gestational age were assessed. The studies probably all employed calibrated scales for birth weight assessment. However, the influence of time before post-delivery weighing on newborn weight recorded in patient files is well recognized.

Fourteen of the 36 studies reported the method for estimating gestational age. Ultrasound fetal measurement was the most common method. Last menstrual period was used together with ultrasound in 3 studies $30,36,37$ and clinical methods were used in 3 studies 2,21,30. Only 5 studies reported more than one method for estimating gestational age, an important procedure for avoiding classification bias 2,30,32,36,37.

Problems with last menstrual period recall, irregular menstruation, oral contraceptive use, and first-trimester bleeding commonly affect the accuracy of gestational age estimation 38 . When compared with last menstrual period, clinical neonatal assessment shows a higher mean overestimation of preterm babies.

Bias can occur in risk estimation during analytical studies on the impact of putative etiological factors for the risk of preterm birth when ultrasound is used. If target exposures interfere with early fetal growth (as suggested with the influence of periodontal disease on preterm birth), then the associated risk of preterm delivery will be overestimated when gestational age is assessed by ultrasound 39 . The validity and precision of ultrasound to estimate gestational age is seriously affected when ultrasound is performed after 18 weeks gestation age and can be an important source of misclassification 39 .

\section{Periodontal disease measurements}

Thirteen different definitions of periodontal disease were used in the 36 selected studies. Furthermore, periodontal disease was assessed by two indices, the CPITN and the Periodontal Disease Index (PDI). CPITN was used in 5 casecontrol studies $6,7,20,23,27$, although it is considered unsuitable for measuring periodontal severity and prevalence in clinical studies. Periodontitis and gingivitis are related but different, and CPITN mixes both and may thus be a source of exposure misclassification. PDI, used by Konopka et al. 22, has similar limitations. The misclassification produced by CPITN and PDI is important, because individuals considered unexposed to periodontal disease can be incorrectly classified as exposed, due to overestimation of periodontal disease, thereby introducing a bias into the analysis.

Because measurement of periodontal disease is so important, it is surprising that only 3 studies provided information on diagnostic reliability in its assessment 11,12,24. Few studies reported the exact percentage of agreement in clinical calibration for periodontal examination 6,26,31,36. However, that method is not an adequate statistical test for analyzing measurement reproducibility.

\section{Confounding}

The most interesting feature observed in all the studies testing the association between periodontal disease and adverse pregnancy outcomes was the inconsistency in controlling for confounders. Psychological stress, physical activity, gestational weight gain, violence, and social support are important risk factors for adverse pregnancy outcomes. Nevertheless, only one study 24 analyzed stress, and the other important risk factors were not taken into account in any study on periodontal disease and adverse outcomes in pregnancy. This is a major shortcoming and raises doubts as to the conclusions of all such studies. 


\section{Statistical issues}

The positive associations between periodontal disease and adverse pregnancy outcomes found in 5 studies may be confounded by the effect of potential variables for adverse pregnancy outcomes, because only bivariate analyses were performed $15,19,23,28,34$. The latter technique is unsuitable for performing statistical inferences, due to lack of control of various confounders (Table 4). In all other studies the statistical analyses were performed using multivariate analysis, which allows determination of the independent contribution of each risk factor to the development of adverse outcomes.

Few confounders were taken into account when multivariate logistic analysis was performed, and no study reported on the data modeling procedures or specified if they had tested whether the model fit the data (residual analysis).

\section{Discussion and conclusions}

Strategies to reduce neonatal and infant mortality due to preterm birth and low birth weight have been supported by evidence-based neonatal medicine. Researchers in periodontal medicine have contributed information on this subject, since studies linking periodontal disease and adverse pregnancy outcomes may help elucidate other risk factors for adverse pregnancy outcomes. In fact, the ultimate goal of dental studies is to find evidence to support dental screening of pregnant women and determine whether treatment of their periodontitis can decrease the risk of adverse pregnancy outcomes.
Although most of the studies analyzed found a positive association between periodontal disease and increased risk of adverse pregnancy outcomes, the methodological limitations raise serious doubts concerning the validity of the outcomes and conclusions. There was considerable variation in methodological quality, with virtually every study showing serious shortcomings, including small sample size, limited number of statistical analyses, inadequate control for potential confounders, inadequate assessment of gestational age and measurement of periodontal disease, and reliance on cross-sectional data.

A meta-analysis was not performed in this systematic review because of the above-mentioned methodological heterogeneity. Metaanalysis is considered a powerful tool to obtain a summary measure of association when systematic reviews are conducted. A recent systematic review found a strong association between periodontal disease and preterm and/or low birth weight 40 . However, the findings were probably biased, since they included only 5 studies compared with the 36 studies analyzed in the present review, suggesting some limitation in their search strategy and inclusion criteria.

A complication in the combined analysis of epidemiological studies in the present review is the diversity of periodontal disease measurements and the lack of consensus on definition and classification of periodontal disease. Robust measurement of periodontal disease should use periodontal pocket depth and clinical attachment level. A pilot study is essential to ensure that examiners are properly calibrated.

Future studies should use more than one method for gestational age estimation. In ante-

\begin{tabular}{ll}
\hline \multicolumn{2}{l}{ Risk factors } \\
\hline Clinical conditions & Social characteristics \\
Individual obstetric history & Mother's age ( 17 or $>35$ years) \\
Multi-fetal pregnancy & Race/ethnicity \\
Placental abnormalities & Low socioeconomic status \\
Smoking & Alcohol abuse \\
Diabetes mellitus & Drug use during pregnancy \\
Vaginal infections & Inadequate prenatal care \\
Immune diseases & Psychological factors \\
Presence of anticardiolipin and lymphocytotoxic antibodies & Certain types of mother's work during pregnancy \\
\hline
\end{tabular}


natal care programs the overall validity of methods to estimate gestational age appears to be high because the vast majority of babies are born at or near term. However, this is misleading in epidemiological studies, because the high percentage of selected preterm births and the gestational age estimation in preterm infants is frequently subject to error.

Extensive literature reviews have highlighted at least 43 potential determinants of adverse pregnancy outcomes (Table 4) 41 . Of the 43 risk factors cited, 11 can be considered confounders in studies on the possible role of periodontal disease in adverse pregnancy outcomes. It is thus vital to control for as many confounding factors as possible by using different strategies like restriction, matching, randomization, and statistical analysis, in order to avoid spurious associations.

The serious methodological limitations of most studies raise serious doubts as to their findings. They do not allow suitable conclusions on the genuine association between periodontal disease and adverse pregnancy outcomes. In conclusion, although 26 of the 36 studies included in this review consider a positive relationship between periodontal disease and adverse pregnancy outcomes, there is no sound scientific justification to recommend screening of periodontal disease in pregnant women as a means to reduce such outcomes.

\section{Resumo}

O objetivo desta revisão sistemática foi avaliar os estudos analíticos que relacionaram a doença periodontal como possível fator de risco para desfechos indesejáveis da gestação. Uma busca bibliográfica foi conduzida nas bases de dados MEDLINE, SciELO, LILACS e Banco de Teses da CAPES em dezembro de 2005. Uma revisão sistemática dos estudos epidemiológicos sobre doença periodontal e desfechos indesejáveis da gestação foi feita. Dentre os 964 estudos identificados, 36 preencheram os critérios de inclusão. Vinte e seis estudos encontraram associações entre a doença periodontal e desfechos indesejáveis da gestação. Observou-se uma heterogeneidade entre os estudos em relação ao método de mensuração na doença periodontal e os desfechos indesejáveis da gestação, não sendo possível realizar uma meta-análise. A maioria dos estudos apresentou falta de controle de variáveis de confusão que tornam suas conclusões duvidosas. Assim como suas limitações metodológicas não permitem adequadas conclusões sobre o real efeito da doença periodontal sobre os desfechos da gestação. Uma possível relação causal permanece desconhecida. Estudos analíticos com maior rigor metodológico, empregando medidas confiáveis para avaliar a exposição e o desfecho serão úteis nas pesquisas futuras.

Doenças Periodontais; Gravidez; Literatura de Revisão

\section{Contributors}

M. V. Vettore designed the methodology, conducted the literature search, and wrote the article. G. A. Lamarca collaborated in the literature search, retrieved references, and participated in the article's final version. A. T. T. Leão helped prepare the methodology and provided orientation for the article. F. B. Thomaz collaborated in the literature search and retrieved references. A. Sheiham provided orientation for the article and participated in writing and editing the final version. M. C. Leal conducted the final revision.

\section{Acknowledgements}

This research was supported by Pan-American Health Organization/World Health Organization (Graduate Thesis Grants in Public Health), the Brazilian National Research Council (CNPq), Carlos Chagas Filho Rio de Janeiro State Research Foundation grants E-26/ 170.421/2003, and Hu-Friedy do Brasil. 


\section{References}

1. Billings F. Chronic focal infections and their etiologic relations to arthritis. Arch Intern Med 1912; 9:484-98.

2. Offenbacher S, Katz V, Fertik G, Collins J, Boyd D, Maynor G, et al. Periodontal infections as a risk factor for preterm low birth weight. J Periodontol 1996; 67:1103-13.

3. Dasanayake AP, Russell S, Boyd D, Madianos PN, Forster T, Hill E. Preterm low birth weight and periodontal disease among African Americans. Dent Clin North Am 2003; 47:115-25.

4. Dasanayake AP, Boyd D, Madianos PN, Offenbacher S, Hills E. The association between Porphyromonas gingivalis-specific maternal serum IgG and low birth weight. J Periodontol 2001; 72:1491-7.

5. Mitchell-Lewis D, Engebretson SP, Chen J, Lamster IB, Papapanou PN. Periodontal infections and pre-term birth: early findings from a cohort of young minority women in New York. Eur J Oral Sci 2001; 109:34-9.

6. Dasanayake AP. Poor periodontal health of the pregnant woman as a risk factor for low birth weight. Ann Periodontol 1998; 3:206-12.

7. Sembene M, Moreau JC, Mbaye MM, Diallo A, Diallo PD, Ngom M, et al. Periodontal infection in pregnant women and low birth weight babies. Odontostomatol Trop 2000; 89:19-22.

8. Louro PM, Fiori HH, Louro Filho P, Steibel J, Fiori RM. Doença periodontal na gravidez e baixo peso ao nascer. J Pediatr (Rio J) 2001; 77:23-8.

9. Moore S, Ide M, Coward PY, Randhawa M, Borkowska E, Baylis R, et al. A prospective study to investigate the relationship between periodontal disease and adverse pregnancy outcome. Br Dent J 2004; 197:251-8.

10. Jarjoura K, Devine PC, Perez-Delboy A, HerreraAbreu M, D'Alton M, Papapanou PN. Markers of periodontal infection and preterm birth. Am J Obstet Gynecol 2005; 2:513-9.

11. Lunardelli AN, Peres MA. Is there an association between periodontal disease, prematurity and low birth weight? A population-based study. J Clin Periodontol 2005; 32:938-46.

12. Moreu G, Tellez L, Gonzalez-Jaranay M. Relationship between maternal periodontal disease and low-birth-weight pre-term infants. J Clin Periodontol 2005; 32:622-7.

13. Cruz SS, Costa MCN, Filho ISGF, Vianna MIP, Santo CT. Maternal periodontal disease as a factor associated with low birth weight. Rev Saúde Pública 2005; 39:782-7.

14. Madianos PN, Lieff S, Murtha AP, Boggess KA, Auten Jr. RL, Beck JD, et al. Maternal periodontitis and prematurity. Part II: Maternal infection and fetal exposure. Ann Periodontol 2001; 6:17582.

15. Hasegawa K, Furuichi Y, Shimotsu A, Nakamura M, Yoshinaga M, Kamitomo M, et al. Associations between systemic status, periodontal status, serum cytokine levels, and delivery outcomes in pregnant women with a diagnosis of threatened premature labor. J Periodontol 2003; 74:1764-70.

16. Goepfert AR, Jeffcoat MK, Andrews WW, Faye-Petersen O, Cliver SP, Goldenberg RL, et al. Peri- odontal disease and upper genital tract inflammation in early spontaneous preterm birth. Obstet Gynecol 2004; 104:777-83.

17. Moore S, Randhawa M, Ide M. A case-control study to investigate an association between adverse pregnancy outcome and periodontal disease. J Clin Periodontol 2005; 32:1-5.

18. Moore S, Ide M, Randhawa M, Walker JJ, Reid JG, Simpson NA. An investigation into the association among preterm birth, cytokine gene polymorphisms and periodontal disease. BJOG 2004; 111:125-32.

19. Offenbacher S, Jared HL, O'Reilly PG, Wells SR, Salvi GE, Lawrence HP, et al. Potential pathogenic mechanisms of periodontitis-associated pregnancy complications. Ann Periodontol 1998; 3:23350.

20. Davenport ES, Williams CE, Sterne JA, Murad S, Sivapathasundram V, Curtis MA. Maternal periodontal disease and preterm low birthweight: case-control study. J Dent Res 2002; 81:313-8.

21. Moliterno LF, Monteiro B, Figueredo CMS, Fischer RG. Association between periodontitis and low birth weight: a case-control study. J Clin Periodontol 2005; 32:886-90.

22. Konopka T, Rutkowska M, Hirnle L, Kopec W, Karolewska E. The secretion of prostaglandin E2 and interleukin 1-beta in women with periodontal diseases and preterm low-birth-weight. Bull Group Int Rech Sci Stomatol Odontol 2003; 45:1828.

23. Carta G, Persia G, Falciglia K, Iovenitti P. Periodontal disease and poor obstetrical outcome. Clin Exp Obstet Gynecol 2004; 31:47-9.

24. Noack B, Klingenberg J, Weigelt J, Hoffmann T. Periodontal status and preterm low birth weight: a case control study. J Periodontal Res 2005; 40: 339-45.

25. Buduneli N, Baylas H, Buduneli E, Turkoglu O, Kose T, Dahlen G. Periodontal infections and preterm low birth weight: a case-control study. J Clin Periodontol 2005; 32:174-81.

26. Cardoso EOC. O estudo das doenças periodontais em gestantes e seu impacto no nascimento de crianças prematuras e/ou de baixo peso [Dissertação de Mestrado]. Rio de Janeiro: Universidade Federal do Rio de Janeiro; 1999.

27. Mokeem SA, Molla GN, Al-Jewair TS. The prevalence and relationship between periodontal disease and pre-term low birth weight infants at King Khalid University Hospital in Riyadh, Saudi Arabia. J Contemp Dent Pract 2004 15; 5:40-56.

28. Dörtbudak O, Eberhardt R, Ulm M, Persson GR. Periodontitis, a marker of risk in pregnancy for preterm birth. J Clin Periodontol 2005; 32:45-52.

29. Radnai M, Gorzó I, Nagy E, Urbán E, Novák T, Pál A. A possible association between preterm birth and early periodontitis. J Clin Periodontol 2004; 31:736-41.

30. López NJ, Smith PC, Gutierrez J. Higher risk of preterm birth and low birth weight in women with periodontal disease. J Dent Res 2002; 81:58-63.

31. Jeffcoat MK, Geurs NC, Reddy MS, Cliver SP, Goldenberg RL, Hauth JC. Periodontal infection 
and preterm birth: results of a prospective study. J Am Dent Assoc 2001; 132:875-80.

32. Offenbacher S, Lieff S, Boggess KA, Murtha AP, Madianos PN, Champagne CM, et al. Maternal periodontitis and prematurity. Part I: obstetric outcome of prematurity and growth restriction. Ann Periodontol 2001; 6:164-74.

33. Holbrook WP, Oskarsdottir A, Fridjonsson T, Einarsson H, Hauksson A, Geirsson RT. No link between low-grade periodontal disease and preterm birth: a pilot study in a healthy Caucasian population. Acta Odontol Scand 2004; 62:177-9.

34. Marin C, Segura-Egea JJ, Martinez-Sahuquillo A, Bullon P. Correlation between infant birth weight and mother's periodontal status. J Clin Periodontol 2005; 32:299-304.

35. Rajapakse PS, Nagarathne M, Chandrasekra KB, Dasanayake AP. Periodontal disease and prematurity among non-smoking Sri Lankan women. J Dent Res 2005; 84:274-7.

36. López NJ, Smith PC, Gutierrez J. Periodontal therapy may reduce the risk of preterm low birth weight in women with periodontal disease: a randomized controlled trial. J Periodontol 2002; 73:911-24.
37. Jeffcoat MK, Hauth JC, Geurs NC, Reddy MS, Cliver SP, Hodgkins PM, et al. Periodontal disease and preterm birth: results of pilot intervention study. J Periodontol 2003; 74:1214-8.

38. Berg AT, Bracken MB. Measuring gestational age: an uncertain proposition. Br J Obstet Gynaecol 1992; 99:280-2.

39. Henriksen TN, Wilcox AJ, Heedegaard M, Secher NJ. Bias in studies of preterm and post-term delivery due to ultrasound assessment of gestational age. Epidemiology 1995; 6:533-7.

40. Khader YS, Ta'ani Q. Periodontal diseases and the risk of preterm birth and low birth weight: a meta-analysis. J Periodontol 2005; 76:161-5.

41. Kramer MS. Determinants of low birth weight: methodological assessment and meta-analysis. Bull World Health Organ 1987; 65:663-737.

Submitted on 30/Aug/2005

Final version resubmitted on $04 / \mathrm{Jan} / 2006$

Approved on 03/Apr/2006 